\title{
A Thermal Model for Electrothermal Simulation of Power Modules
}

\author{
Jinlei Meng *, Xuhui Wen *, Yulin Zhong *, and Zhijie Qiu *
}

\begin{abstract}
A thermal model of power modules based on the physical dimension and thermal properties is proposed in this paper. The heat path in the power module is considered as a one-dimensional heat transfer in the model. The method of the parameters extraction for the model is given in the paper. With high speed and accuracy, the thermal model is suit for electrothermal simulation. The proposed model is verified by experimental results.
\end{abstract}

Keywords: Power module, Junction temperature, Thermal resistance, Thermal capacitance, Thermal time constant

\section{Introduction}

With the development of the semiconductor manufacture technology, the power electronic chips of the power modules become high voltage and high power with a decreasing of chip size, resulting a increasing power density of power modules. Therefore, the junction temperature $\left(T_{j}\right)$ of the chip is a more and more important parameter for the reliability and stability of the power module operations. Generally, a limit maximum junction temperature is given in the datasheet to keep the operation within the Safe Operation Area (SOA). In order to avoid over temperature destroy of power modules, a correct junction temperature should be achieved.

The junction temperature $T_{j}$ is determined by the ambient temperature, the power loss of power module, thermal resistance from junction to case $\left(R_{t h j c}\right)$ and the heatsink configuration. Within these aspects above, ambient temperature and power loss are dependent on the application requirement; thermal resistance is determined by structure and materials of power modules.

Power loss of power modules is mainly dissipated by the path from junction to case and then through heatsink to ambient. Temperature rise of the junction consists of two series parts: one is the temperature rise from junction to case which is the product of power loss and thermal resistance from junction to case $\left(R_{t h j c}\right)$, and the other is the temperature difference between case and heatsink which

\footnotetext{
* University of Chinese Academy of Sciences, Chinese Academy of Sciences, Beijing Engineering Laboratory of Electrical Drive System \& Power Electronic Device Packaging Technology, Beijing, China (mengjinlei@mail.iee.ac.cn,wxh@mail.iee.ac.cn, zhongyulin@mail. iee. ac.cn, qiuzhijiei@mail.iee.ac.cn)

Received 3 October 2013; Accepted 20 October 2013.
}

mainly depends on the configuration of cooling system. Thermal characteristics of power modules are the main tasks to simulate in this paper.

There are two main methods to simulate thermal characteristics of power modules: Finite Element Method (FEM) and Resistance Capacitance (RC) equivalent circuit. FEM is a numeric method simulating the relationship between thermal responses and heat resource (power loss) by a large number of finite elements. And RC model characters the relationship by thermal resistance and thermal capacitance. FEM is more accurate than RC model but consumes much more time [1]. RC is more widely used because it is simple and accomplished easily in general circuit simulation software [2].

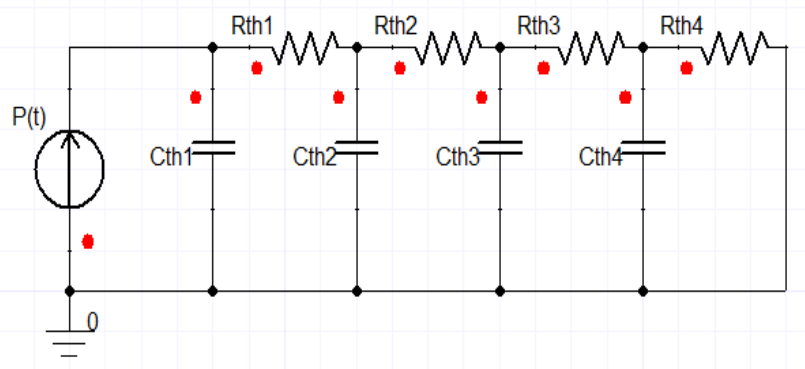

Fig. 1. RC thermal model of power modules

Besides, RC model is easy to extend to electrothermal simulation [3] of power modules. However, $R_{t h i}$ and $C_{t h i}$ in RC model are generally exacted from thermal impendence response curve of measurement [4]-[5] or FEM and, therefore RC model can't be achieved independently. To overcome the drawback of the RC and keep its advantage, a thermal simulation model is given in this paper and the proposed method is independent. 


\section{Present Thermal Models for the Power Module}

Heat transfer consists of three types of process modes: heat conduction through a solid or a stationary fluid, heat convection from a surface to a moving fluid, net radiation heat exchange between two surfaces without any medium [6]. Among these three modes, heat conduction plays the most important part within heat transfer of power modules.

\subsection{Finite Element Method}

The finite element method simulates the thermal characteristics of power modules by solving heat transfer equation of a large number of elements. Therefore, heat transfer equation and element meshing are the main parts of FEM.

According to the heat transfer theory, the three dimensional heat conduction in a solid is described with principle of energy balance and governed by (1) as follows:

$$
\rho c_{p} \frac{\partial T}{\partial t}=\lambda \nabla^{2} T+q
$$

here, $T$ refers to temperature of the objective, $\mathrm{t}$ is the time variable, $c_{p}, \rho, \lambda$ are the specific heat, density and thermal conductivity of the material, respectively, and $q$ is heat generated in the solid.

FEM solves (1) for all elements of power modules with FEM software after the meshing operation, shown in Fig. 2. Nodes of elements are assigned with boundary condition and temperature distribution of power modules are achieved after solve operation is executed, shown in Fig. 3.

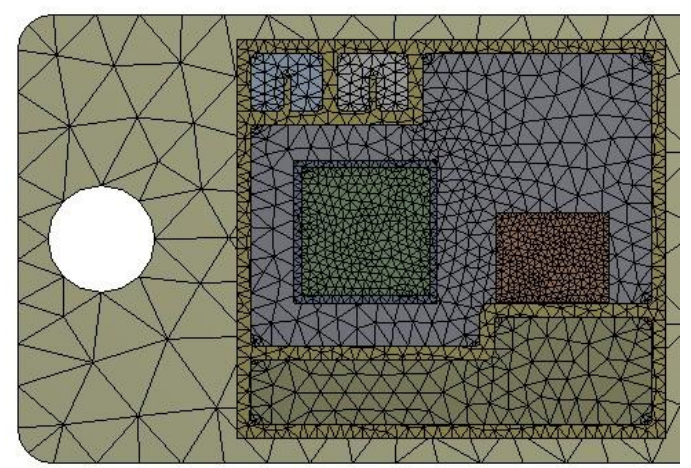

Fig. 2. FEM elements of power module after meshing operation

\subsection{Resistance Capacitance Equivalent Circuit}

$\mathrm{RC}$ thermal model considers the heat transfer path from chip to baseplate of the power module as a series of resistance and capacitance unit. RC models are classified into two types: Foster style and Cauer. Foster RC model consists of parallel resistance and capacitance unit in series and is also called series style, illustrated in Fig. 4. Cauer RC model consists of capacitance and resistance unit and sub RC model in parallel and is also called parallel style, shown in Fig. 1.

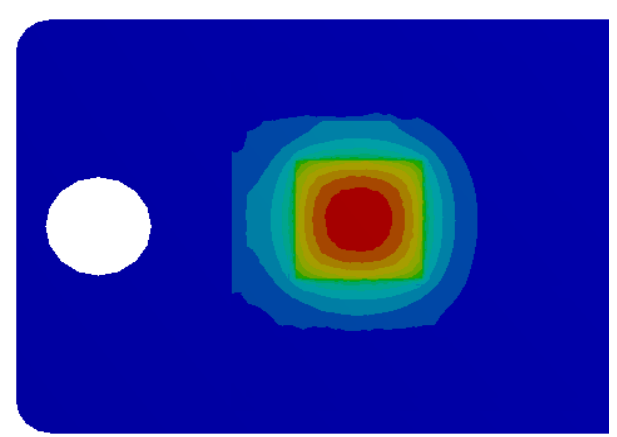

Fig. 3. Temperature distribution of power module with FEM

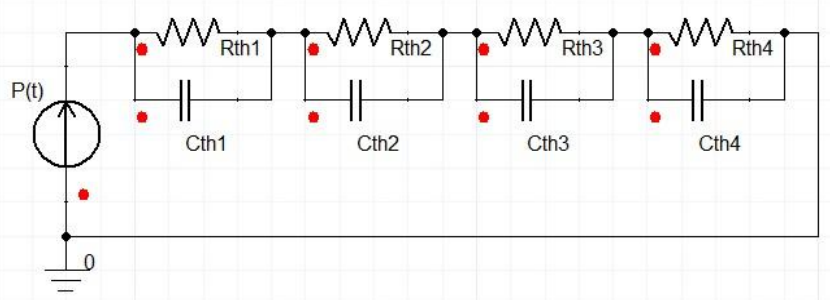

Fig. 4. RC model of power modules(Foster)

Generally, RC model is accomplished by fitting the thermal impendence curve measured by experiment or FEM simulation [4]. For the Foster RC model, the Rthi and Cthi are curve fitted by the equation as follows:

$$
Z_{t h j c}=\sum_{i=1}^{n} R_{t h i}\left(1-e^{-t / \tau_{i}}\right), \tau_{i}=R_{t h i} C_{t h i}
$$

here, $Z_{\text {thjc }}$ refers to thermal impendence from junction to case of power modules, $\tau_{\mathrm{i}}$ is the thermal response constant of $i^{\text {th }} \mathrm{RC}$ unit and $R_{t h i}, C_{t h i}$ are thermal resistance and thermal capacitance, respectively. It is worth noting that Foster RC model is only a mathematic expression of curve fitting without any physical meaning. Cauer RC model stands for the actual physical mechanical of structure layers. Cauer and Foster are used for different applications and could be transfer to each other easily [7].

FEM gives a high accuracy but consumes time heavily. $\mathrm{RC}$ model has to get the model parameters from other data and lacks of independent. A accuracy method with speed is required in thermal simulation and in the optimizing process of power modules. 


\section{Principle of the Proposed Method}

\subsection{One-Dimensional Heat Transfer Theory}

Since the thickness is much less than length and width of each layer, the heat flow path from chip to case of power module can be considered as one-dimensional (1-D) heat transfer as Fig. 5, according to the heat transfer theory. The thermal resistance of 1-D heat transfer can be calculated by the equation as follows:

$$
R_{t h}=d /(\lambda A)
$$

here $d$ is heat conduct length along the heat flow direction, $A$ is effective cross area perpendicular to the heat flow direction and $\lambda$ is thermal conductivity of the material. The thermal capacitance of the RC model is calculated by:

$$
C_{t h}=c_{p} \rho V,
$$

here $c_{p}, \rho$ are the specific heat and density of the material, $V$ is the effective volume of the heat transfer in power modules.

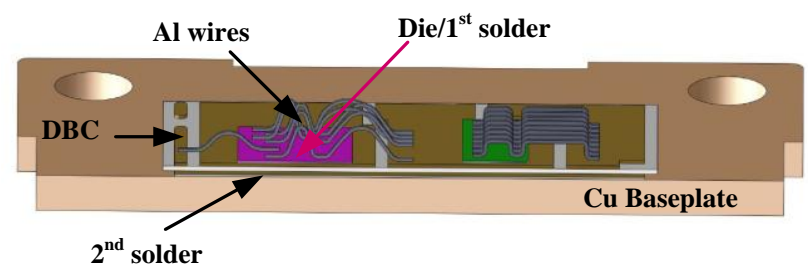

Fig. 5. 3-D cross section of power modules (FS450R12KE4)

For a certain power loss condition, the steady-state temperature rise of junction to case and response speed are determined by the value of Rth and Cth, governed by (5):

$$
\Delta T_{j c}= \begin{cases}P \cdot R_{t h j c} & \text { for Steady-state } \\ P \cdot R_{t h j c}\left(1-e^{-t / \tau}\right) & \text { for Trans.-state }\end{cases}
$$

where $P$ is the power loss flow from chip to case, produced by the electrical operations, Rthjc is the thermal resistance from junction to case, $\tau$ is a heat transfer time constant determined by Rthjc and Cthjc.

\subsection{Parameters Determination}

It's an important issue to extract $A$ and $V$ in (3) and (4) which determine accuracy of the thermal model. As the physical dimension of structure layers increases along the heat flow direction in the power module, the effective cross area from junction to case spread to a larger value of $A$ especially in the baseplate (case). According to the heat transfer theory, $A$ is determined by the equations:

$$
\begin{aligned}
& A_{e f f 2}=\left(L_{1} W_{1}\right)\left(L_{2} W_{2}\right) / 2, \\
& \left\{\begin{array}{l}
L_{2}=L_{1}\left(1+2 \tan \alpha_{1,2}\right), \\
W_{2}=W_{1}\left(1+2 \tan \alpha_{1,2}\right),
\end{array}\right.
\end{aligned}
$$

where $\alpha$ is spread angle demonstrated in Fig. 6. And the spread angle is governed by the equation as follows:

$$
\alpha_{1,2}=\arctan \left(\lambda_{1} / \lambda_{2}\right)
$$

and $V$ is the volume of the heat flow through each physical structure in power modules, given by the equation as follows:

$$
V_{\text {eff } 2}=A_{e f f 2} d_{2}
$$
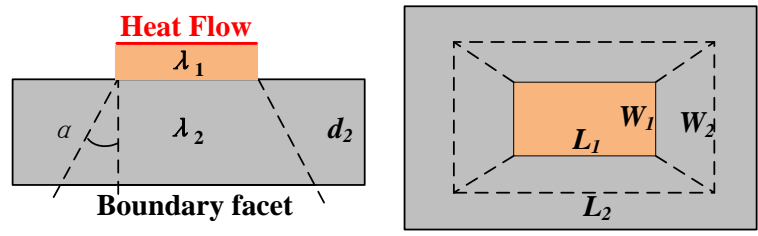

Fig. 6. Extraction of $\mathrm{A}$ and $\mathrm{V}$ for thermal model of power modules

\section{Realization and Verification}

\subsection{Realization Based on Commercial IGBT Power Module}

The thermal resistance from junction to case is achieved by the present method based on the physical structure of commercial IGBT power modules MMG50S120B6TN and FS450R12KE4. The parameters $d$ is comes from the physical dimensions of the two power modules, and $\mathrm{A}, \mathrm{V}$ are determined by d, $\alpha$ of each layer based on the method in Section 3.2.

As a power electronic module consists of 7 layers of die, 1st solder, DBCs (copper-ceramic-copper), 2nd solder and baseplate copper, shown in Fig. 5, a RC equivalent circuit with 7 orders can be achieved for a power module in MATLAB m-file. The results Rthi and Cthi are sent to 
Simulink, demonstrated in Fig. 7 and Table 1. It must be noted that each phase half bridge of FS450R12KE4 is packaged by three same DBC units in parallel. Consequently, results of the power module should be divided by 3 after RC model simulation in Fig. 7 .

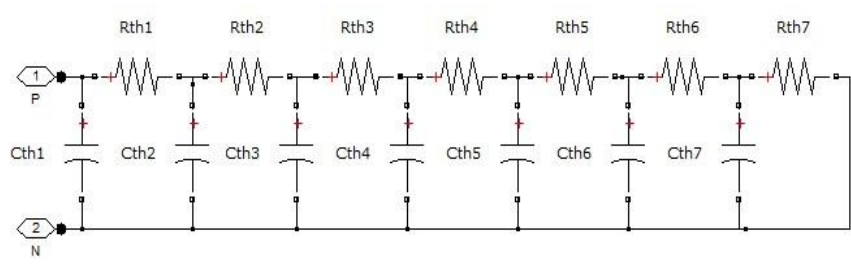

Fig. 7. RC model of a power module from junction to case

Table 1. Thermal resistance and thermal capacitance of power modules

\begin{tabular}{|c|c|c|c|c|}
\hline & \multicolumn{2}{|c|}{ FS450R12KE4 } & \multicolumn{2}{c|}{ MMG50S120B6TN } \\
\hline $\begin{array}{c}\text { Layer } \\
\text { No.i }\end{array}$ & $\begin{array}{c}R_{t h i} \\
(\mathrm{~K} / \mathrm{W})\end{array}$ & $\begin{array}{c}C_{t h i} \\
(\mathrm{~J} / \mathrm{K})\end{array}$ & $\begin{array}{c}R_{t h i} \\
(\mathrm{~K} / \mathrm{W})\end{array}$ & $\begin{array}{c}C_{t h i} \\
(\mathrm{~J} / \mathrm{K})\end{array}$ \\
\hline 1 & 0.0048 & 0.0232 & 0.0099 & 0.00805 \\
\hline 2 & 0.0233 & 0.0205 & 0.0576 & 0.0081 \\
\hline 3 & 0.0051 & 0.1548 & 0.0117 & 0.0543 \\
\hline 4 & 0.0808 & 0.2061 & 0.1276 & 0.04535 \\
\hline 5 & 0.0042 & 0.1865 & 0.0095 & 0.06645 \\
\hline 6 & 0.0490 & 0.0577 & 0.0784 & 0.0149 \\
\hline 7 & 0.0373 & 2.116 & 0.0666 & 0.5926 \\
\hline
\end{tabular}

\subsection{Verification by Experiment}

A series of experiments are finished by thermal resistance test equipments (shown in Fig. 8 and Fig. 9), which is based on the principle of Electrical Temperature Sensitive Parameters (ETSP) method. The method is realized through a sensitive parameter representing the relationship between junction temperature and the voltage drop across anode and cathode (Vce) of the power module. Once a current is applied to the power module, a Vce value is measured and the junction temperature is confirmed. It is worth noting that the temperature measured is an average of the test current of the power module, which is a little lower than the maximum junction temperature of the chips.

The thermal resistances of two power modules are measured at the condition as below: Ambient temperature $\mathrm{Ta}=70^{\circ} \mathrm{C}$, Gate driver voltage Vggon $=15 \mathrm{~V}$, Measure current Itest $=100 \mathrm{~mA}$, Heating current Iheat $=100 \mathrm{~A}$ and $30 \mathrm{~A}$ for FS450R12KE4 and MMG50S120B6TN, respectively.

The measured thermal impendence results of ETSP method are shown in Fig. 10 and Fig. 11, the results of the proposed method are given simultaneously. The results of proposed method agree with the experiment data, however, with much less complexity and less time consuming than FEM.

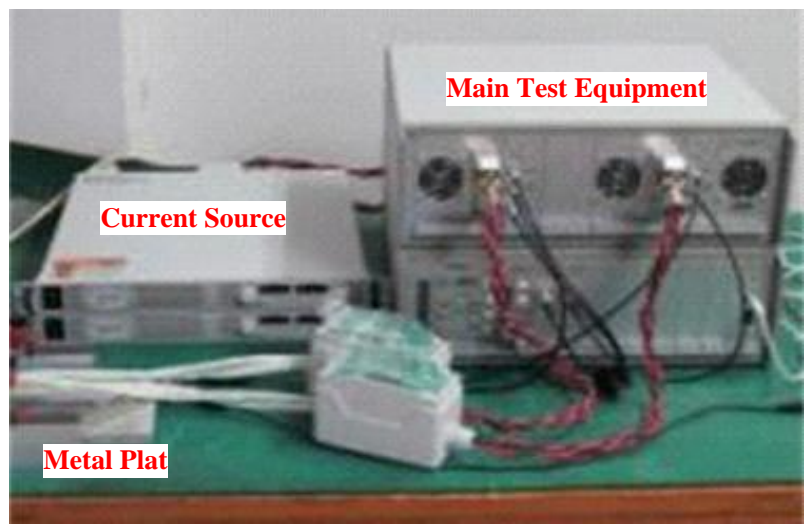

Fig. 8. Thermal resistance test equipments

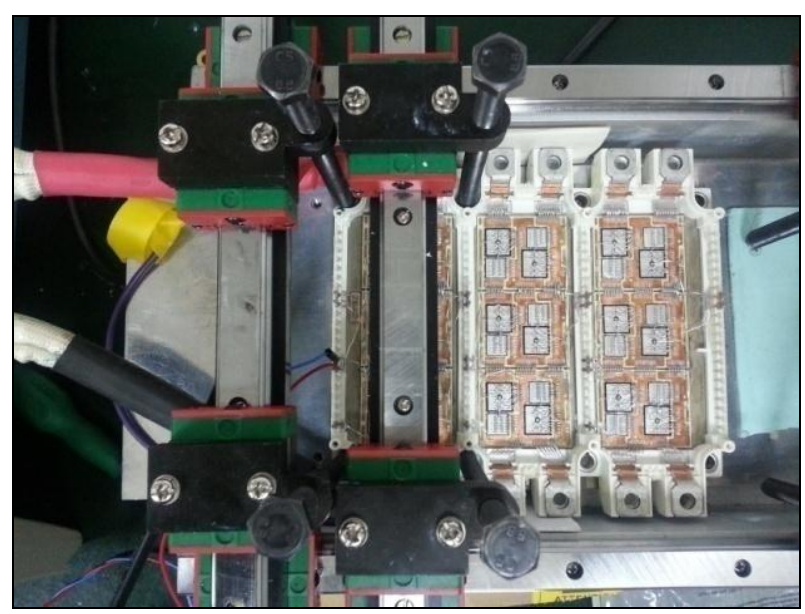

Fig. 9. FS450R12KE4 under test

Thermal characteristics of power modules consists of two most important parameters: the steady state thermal response characteristic $R_{t h j c}$, also named thermal resistance, and the thermal response time constant $\tau_{t h}$. Comparisons of these two parameters between results of the proposed method and the experiment are listed in Table 2.

Results of the proposed method agree with experiment well. The maximum error are $2.7 \%$ of $R_{t h j c}$ and $31 \%$ of $\tau_{t h}$. And the proposed method is easy to accomplish and much less time cost than FEM.

The RC model might be extended to electro-thermal model in any circuit simulation software easily only with a electrical-thermal interface. Power loss and chip temperature are the two parameters required in the interface.

The nonlinearity effect of temperature on thermal model can be taken in by dividing each layer structure into several sublayers with uniform temperature internally. 


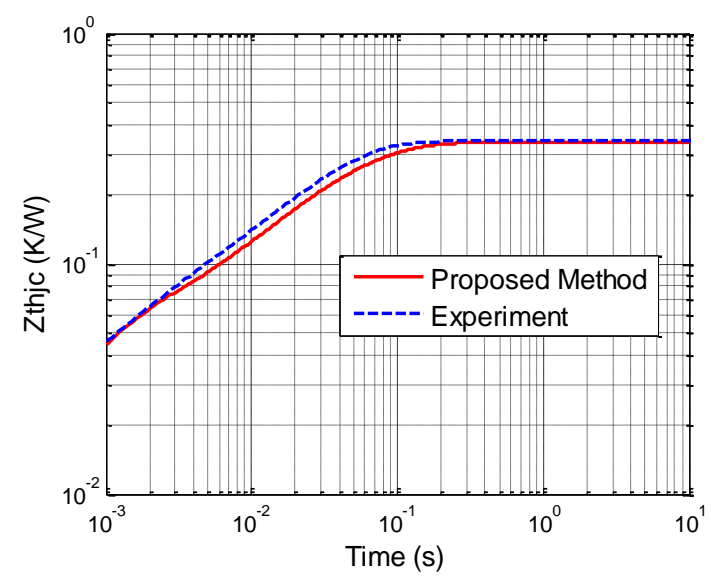

Fig. 10. Comparison between the proposed method and experiment for MMG50S120B6TN

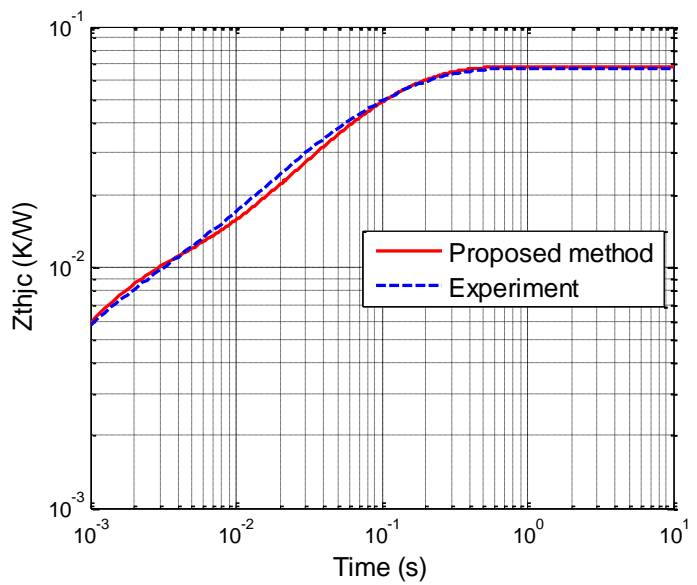

Fig. 11. Verification of the proposed method of FS450R12KE4

Table 2. Comparison of thermal resistance and thermal response constant time

\begin{tabular}{|c|c|c|c|c|c|c|}
\hline \multirow{2}{*}{$\begin{array}{c}\text { Power } \\
\text { Module }\end{array}$} & \multicolumn{3}{|c|}{$\begin{array}{c}\text { Thermal resistance } \\
\text { (K/W) }\end{array}$} & \multicolumn{3}{c|}{$\begin{array}{c}\text { Thermal response } \\
\text { time constant (ms) }\end{array}$} \\
\cline { 2 - 7 } & Exp & $\begin{array}{c}\text { Proposed } \\
\text { method }\end{array}$ & $\begin{array}{c}\text { Error } \\
(\%)\end{array}$ & Exp & $\begin{array}{c}\text { Proposed } \\
\text { method }\end{array}$ & $\begin{array}{c}\text { Error } \\
(\%)\end{array}$ \\
\hline $\begin{array}{c}\text { MMG50S1 } \\
\text { 20B6TN }\end{array}$ & 0.342 & 0.335 & -2 & 26.7 & 35.0 & +31 \\
\hline $\begin{array}{c}\text { FS450R1 } \\
\text { 2KE4 }\end{array}$ & 0.0667 & 0.0685 & +2.7 & 64.5 & 71.5 & +11 \\
\hline
\end{tabular}

${ }^{a}$ Relative error of results of the proposed method to that of experiment

\section{Conclusion}

Based on physical dimension and thermal properties of material in power modules, a thermal model is proposed and analyzed. The thermal model provides a junction temperature estimation method for electro-thermal simulation of power modules with high speed and accuracy. Nonlinearity effect of temperature on thermal resistance can be taken into the proposed method if necessary. Both steady and transient states are verified by experiment.

\section{Acknowledgements}

This work is supported by National Science and Technology Support Program 2013BAG02B00: EMC Design \& Application for Electric Vehicles Driving System and National Science and Technology Major Project: 2011ZX02603-003.

\section{References}

[1] S. Wen, and L. Guo-Quan, "Finite-element modeling of thermal and thermomechanical behavior for threedimensional packaging of power electronics modules," in Thermal and Thermomechanical Phenomena in Electronic Systems, 2000, pp. 303-309.

[2] Z. Luo, A. Hyungkeun, and M. A. E. Nokali, "A thermal model for insulated gate bipolar transistor module," IEEE Transactions on Power Electronics, vol. 19, pp. 902-907, 2004.

[3] Z. Z. a. P. Igic, "High-speed electro-thermal modelling of a three-phase insulated gate bipolar transistor inverter power module," International Journal of Electronics, vol. 97, pp. 11, February 2010.

[4] C.-S. Yun, P. Malberti, M. Ciappa, and W. Fichtner, "Thermal component model for electrothermal analysis of IGBT module systems," IEEE Transactions on Advanced Packaging, vol. 24, pp. 401-406, 2001.

[5] T. Kojima, Y. Yamada, M. Ciappa, M. Chiavarini, and W. Fichtner, "A novel electro-thermal simulation approach of power IGBT modules for automotive traction applications," Proceedings in Power Semiconductor Devices and ICs, 2004. 2004, pp. 289-292.

[6] T. L. BERGMAN, A. S. LAVINE, F. P. INCROPERA, and D. P. DEWITT, "Fundamentals of heat and mass transfer," SEVENTH EDITION ed. USA: John Wiley \& Sons, Inc., 2011.

[7] Y. C. Gerstenmaier, W. Kiffe, and G. Wachutka, "Combination of thermal subsystems modeled by rapid circuit transformation," in Thermal Investigation of ICs and Systems, 2007, pp. 115-120.

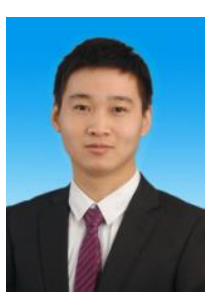

Jinlei Meng received the B.S. degree in Automation from Huazhong University of Science and Technology, Wuhan, China, in 2009. He has been a Ph.D student in Institute of Electrical Engineering, Chinese Academy of Sciences, Beijing, China, since 2009, majoring in Power Electronics and Power Drive. His research interests include characterization, modeling, and packaging of power semiconductor devices. 


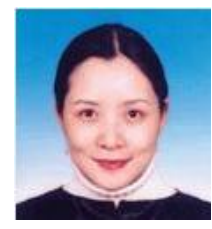

Xuhui Wen received the B.S., M.S., and $\mathrm{Ph} . \mathrm{D}$. degrees in electrical engineering from Tsinghua University, Beijing, China, in 1984, 1987, and 1993, respectively. Since 1993, she has been with the Institute of Electrical Engineering (IEE), Chinese Academy of Sciences (CAS), Beijing, China, where she became an Associate Professor in 1996 and a Professor in 1999. From 2004 to 2005, she was a Visiting Researcher with The Ohio State University. Her research fields include power electronics, motion control, and electric vehicle drives.

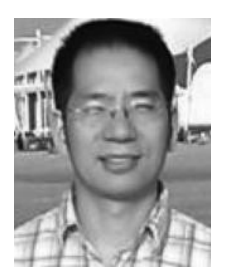

Yulin Zhong was born in Anhui, China. He received the B.Sc. degree from Xi'an Jiaotong University, Xi'an, China, in 1996, and the M.Sc. and Ph.D. degrees from Tsinghua University, Beijing, China, in 2005 and 2009, respectively.

From 1996 to 2002, he worked first as an Electrical Engineer and later as a Software Engineer in Guangdong, China. He is currently an Assistant Researcher at the Institute of Electrical Engineering, Chinese Academy of Sciences, Beijing. His main research interests include power converter for electrical vehicles, electromagnetic compatibility design for power converters, and packaging development for high power semiconductor modules.

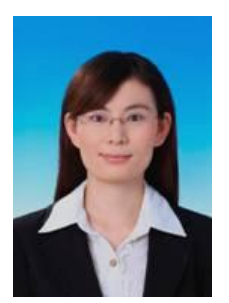

Zhijie Qiu received the B.S. in Optoelectronics and Information Engineering from Beihang University, China, in 2009, and the M.S. in Reliability and Systerm Engineering from Beihang University, China, in 2012. The research interest and expertise is in electronic reliability and reliability physics. She is currently a research assistant in Institute of Electrical Engineering, Chinese Academy of Sciences. Her research interests include IGBT modules thermal measurement and thermal-mechanical fatigue failure. She is also involved in the field of reliability and lifetime evaluation of IGBT modules. 